\title{
1 A Randomized, Placebo-Controlled Trial of Beloranib for the Treatment of Hypothalamic Injury-
}

\section{Associated Obesity}

3 Authors: Ashley Shoemaker $\mathrm{MD}^{1}$, Joseph Proietto MB BS, $\mathrm{PhD}^{2}$, M. Jennifer Abuzzahab $\mathrm{MD}^{3}$, Tania

4 Markovic $\mathrm{MD}^{4}$, Jaret Malloy $\mathrm{PhD}^{5}$, Dennis D Kim $\mathrm{MD}^{5}$

5

$6 \quad{ }^{1}$ Vanderbilt University, Nashville, TN

$7 \quad{ }^{2}$ University of Melbourne, Department of Medicine, Austin Health, Heidelberg, Australia

$8{ }^{3}$ Children's Hospitals and Clinics of Minnesota, St. Paul, MN

$9{ }^{4}$ Boden Institute of Nutrition, Exercise \& Eating Disorders, University of Sydney, Sydney Australia

$10 \quad{ }^{5}$ Zafgen Inc., Boston, MA

11

12 Running Title: Beloranib for HIAO

13 Word count abstract: 244

14 Word count main text: 3512

15 Number of figures and tables: 3

16 Number of references: 28

17

18 Clinical Trial Registration: NCT02063295

19

20 Corresponding Author (same as address for reprints):

21 Dennis Kim

22 Zafgen, Inc., 175 Portland St, $4^{\text {th }}$ Floor

23 Boston, MA 02114

24 This is the author manuscript accepted for publication and has undergone full peer review but has not been through the copyediting, typesetting, pagination and proofreading process, which

25858 3144.5789differences between this version and the Version of Record. Please cite this article 26 as doi: $10.1111 /$ dom.12928

This article is protected by copyright. All rights reserved. 


\section{ABSTRACT}

28 Aims: Hypothalamic injury-associated obesity (HIAO) results from damage to the hypothalamus that

29 often occurs with surgical removal/radiation therapy of tumors in the hypothalamic region, such as

30 craniopharyngioma. There is currently no rigorously studied pharmaceutical treatment for the intractable

31 weight gain and cardiometabolic consequences that occur in patients with HIAO. We aimed to assess

32 efficacy, safety, and tolerability of beloranib treatment for 4 to 8 weeks in patients with HIAO.

33 Materials and Methods: This Phase 2a, double-blind, placebo-controlled study included 14 patients with

34 HIAO randomized to receive beloranib $1.8 \mathrm{mg}$ or placebo subcutaneously twice weekly for 4 weeks with

35 an optional 4-week open-label extension in which all patients received beloranib. The primary endpoint

36 was change in weight from baseline to Week 4.

37 Results: Participants were 64\% female, with mean (SD) age 32 (9) years, BMI 43 (7) kg/m², and weight

$38126(22) \mathrm{kg}$. Compared with placebo $(\mathrm{N}=4)$, beloranib $1.8 \mathrm{mg}(\mathrm{N}=8)$ resulted in a mean $(95 \% \mathrm{CI})$

39 difference in weight of $-3.2(-5.4,-0.9) \mathrm{kg}$ after 4 weeks. Weight loss continued through the 8 weeks in

40 patients randomized to beloranib (mean $-6.2[-8.2,-4.1] \mathrm{kg}$ ). Beloranib treatment was associated with

41 improvements in high-sensitivity CRP. Adverse events were mild to moderate. No patients who received

42 beloranib discontinued treatment.

43 Conclusion: Beloranib treatment resulted in progressive weight loss in patients with HIAO that was

44 comparable to that observed with beloranib in patients with exogenous obesity. These findings indicate a

45 novel mechanism for treating obesity in patients with HIAO.

46 
Hypothalamic injury-associated obesity (HIAO) is a life-altering complication resulting from

49 damage to the hypothalamus. The most common etiology of HIAO is craniopharyngioma, but it is

50 observed with other insults to the hypothalamus [1]. Despite craniopharyngioma having high survival

51 rates, post-treatment pituitary hormone deficiencies and visual and neurological deficits are common [2],

52 and at least $50 \%$ experience intractable weight gain resulting in severe obesity. Patients with HIAO are at

53 increased risk for metabolic and cardiovascular disorders and have increased obesity-associated morbidity

54 and mortality [2, 3].

Treatment options for patients with HIAO are limited. One potential barrier to treatment of HIAO

56 is that most available pharmaceutical agents have putative actions mediated through the hypothalamus.

57 Attempts at caloric restriction or pharmacotherapy have been met with little or only brief success [4, 5].

58 Even bariatric surgery has shown mixed success [6] and is controversial due to unproven efficacy and

59 significant morbidity and mortality [7]. There are no rigorously studied, accepted pharmacological

60 treatments for obesity in patients with HIAO [2].

Methionine aminopeptidase 2 (MetAP2) inhibitors are a novel class of compounds that lead to

62 marked weight loss in animals and humans $[8,9]$. Although not fully elucidated, the mechanism for

63 weight loss associated with MetAP2 inhibitors is believed to be due to a reduction in fat biosynthesis and

64 increased fat oxidation and lipolysis, resulting in reduced fat mass as well as a reduction in caloric intake

65 [9-13]. As patients report a reduction in hunger [9], it is also possible that some MetAP2 inhibitory effects

66 are centrally mediated, or occur through peripheral feedback to the brain, though a mechanism has not

67 been elucidated.

In a Phase 2 clinical trial in patients with exogenous obesity, administration of the MetAP2

69 inhibitor, beloranib, for 12 weeks produced a dose-dependent reduction in body weight (up to

70 approximately 11\%), improvements in markers of cardiometabolic risk, and a reduction in hunger [9]. In

71 obese mice with chemically-induced HIAO with gold-thioglucose, beloranib administration consistently 
72 reduced food intake, body weight, and adipocyte mass. Thus, MetAP2 inhibition may induce weight loss

73 in humans with compromised hypothalamic regulation of energy balance, such as patients with HIAO.

74 Development of beloranib has ceased due to an imbalance in venous thromboembolic events in beloranib-

75 treated patients with obesity and Prader-Willi syndrome (manuscript in preparation) or type 2 diabetes

76 (T2D; manuscript in preparation). Investigation of other MetAP2 inhibitors with similar efficacy but an

77 improved safety margin to mitigate risk of venous thrombotic events is underway.

78 The aim of this clinical trial was to investigate the efficacy, safety, and tolerability of beloranib

79 suspension compared to placebo, administered subcutaneously twice weekly, for 4 weeks in 14 adult

80 patients with HIAO. The clinical trial included an open-label extension (OLE) in which all patients

81 received beloranib for an additional 4 weeks.

82 
This was a double-blind, randomized, placebo-controlled, parallel-group, 4-week study with an optional 4-week OLE. Eligible patients were enrolled at 2 sites in the United States and 2 sites in

87 Australia, between June and September 2014. The Institutional Review Boards at all study sites approved

88 the protocol prior to study initiation. All patients provided written informed consent. Study drug

89 administration could be stopped at any time at the discretion of the Investigator.

91 Response System in a 4:3 ratio to beloranib $(1.8 \mathrm{mg})$ or placebo for the 4-week randomized treatment

92 period. Patients had the option to participate in a subsequent 4-week OLE during which all patients

93 received beloranib $1.8 \mathrm{mg}$ twice weekly.

Blinding was maintained by study Investigators, study staff, and patients throughout the study,

95 except by select pharmacy and designated study drug administrators who did not take part in any other

96 aspects of the study. Beloranib or placebo was administered twice weekly at the study site by a study drug

97 administrator or at the patient's home/work by a home health nurse (study drug administrators were

98 unblinded and were not otherwise involved in the care or evaluation of study subjects) via subcutaneous

99 injection in the lateral abdominal region. Beloranib was prepared by adding diluent (placebo) containing

100 carboxymethylcellose, mannitol, poloxamer 188, and phosphate buffer (pH 7.3) to a single-use vial of

101 beloranib sterile powder. Due to slight differences in coloration (study drug is opaque and placebo is

102 clear) patient blinding was maintained by shielding study drug from the patient's view during the

103 administration process.

105 after the last dose of beloranib or placebo. 
The study included male and female patients aged 18-65 years with obesity as a consequence of

108 acquired anatomical hypothalamic damage as evidenced by medical history and radiographic evidence of 109 hypothalamic lesion on review of magnetic resonance imaging (MRI). All patients had a minimum of

$11010 \%$ self-reported weight gain within 2 years of diagnosis (considered by the Investigator to be secondary

111 to hypothalamic injury), and body mass index (BMI) e 30 and $\mathrm{d} 60 \mathrm{~kg} / \mathrm{m}^{2}$. Additional inclusion criteria are

112 detailed in Supplemental Table 1.

\section{Study Outcomes}

114 The primary outcomes were change in body weight from baseline to Week 4, safety and

115 tolerability. Secondary outcomes included the change in fasting lipids, fasting glucose, self-reported sense

116 of hunger and eating behavior, quality of life, and sleep quality. Exploratory analysis was conducted on

117 biomarkers of lipid metabolism as well as on panels of biomarkers related to cardiometabolic health

118 (Metabolic MAP and Cardiovascular MAP; Myriad RBM, Inc. Austin, TX).

119 Body weight was measured after an overnight fast using a designated calibrated scale. Patients

120 wore a hospital gown and voided immediately prior to being weighted. Fasting hemoglobin A1c

121 (HbA1c), glucose, and insulin levels were measured in patients with T2D. Sense of hunger and eating

122 behavior over the 2 days prior to select study visits were assessed in the fasted state using an 8-Question

123 visual analog scale (8Q-VAS) [14]. Quality of life was assessed using the Impact of Weight on Quality of

124 Life Questionnaire-Lite Version (IWQOL-Lite) [15]. Sleep quality was evaluated using a 3-Question

125 Sleep Quality Questionnaire which assesses sleep latency, duration of sleep, and overall sleep quality. Safety evaluations included assessment of treatment-emergent events (AEs) and serious AEs,

127 physical examination, electrocardiogram, vital signs, concomitant medications, and laboratory

128 parameters. 
The sample size was selected to provide preliminary data and was not based on statistical

131 considerations. The safety population included all participants who received at least 1 dose of blinded

132 study drug. The per protocol (PP) population included all randomized patients who received at least 7 of 8

133 doses of blinded study drug with no major protocol deviations and no dosing errors during the

134 randomized period. The modified intent-to-treat (mITT) population included all randomized patients who

135 received at least 1 dose of blinded study drug and who had at least 1 post-baseline body weight

136 measurement during the randomized treatment period.

137 The primary comparison was the least squares (LS) mean treatment effect between beloranib and

138 placebo at Week 4 in the PP population. Unless otherwise noted, all efficacy data are presented for the PP

139 population. The primary analysis was conducted using an analysis of covariance (ANCOVA) model with

140 absolute or percent change in body weight as the dependent variable, treatment group as a factor, and

141 baseline value as a covariate. Secondary outcomes were tested using an ANCOVA model with treatment

142 group as factors and baseline values as covariates. A 2 -sided test at the $5 \%$ level was used to determine

143 statistical significance. AEs were summarized by treatment group, system organ class, and preferred term.

144

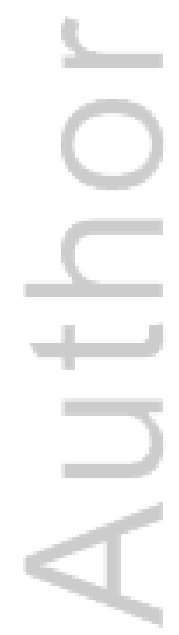


A total of 14 patients were randomized to beloranib $(\mathrm{N}=8)$ and placebo $(\mathrm{N}=6)$ with 13 patients

147 (beloranib $\mathrm{n}=8$; placebo $\mathrm{n}=5$ ) completing the randomized treatment period and the OLE (Supplemental

148 Figure 1). One patient in the beloranib group missed a single dose; there were no other issues with study

149 drug compliance. During the randomized treatment period, 1 patient discontinued placebo treatment after

1503 doses due to an AE of urticaria and 1 additional patient receiving placebo treatment was excluded from

151 the PP population prior to data analysis due to receiving a dose of active drug.

Patient baseline demographics and characteristics were generally similar across treatment groups

153 (Table 1$)$. The majority of patients were white (78.6\%) females $(64.3 \%)$, with a mean age of 31.9 (range

15418 to 48 ) years, mean BMI of 42.9 (range 30.3 to 54.8 ) $\mathrm{kg} / \mathrm{m}^{2}$ and mean body weight $126.4 \mathrm{~kg}$ (range 89.7

155 to 166.3 ). The study included 3 patients with T2D (2 beloranib, 1 placebo). No patients were treated with

156 glucagon-like peptide-1 (GLP-1) receptor agonists.

\section{Body Weight} patients randomized to beloranib but remained stable for patients randomized to placebo (Figure 1 and Supplemental Figure 2). At Week 4, the change in body weight in the PP population was statistically greater for patients randomized to beloranib compared with placebo (LS mean [95\% Confidence Interval] difference from placebo: $-3.2 \mathrm{~kg}[-5.4,-0.9 ; \mathrm{p}=0.010] ;-2.6 \%$ change from baseline $[-4.5,-0.7 ; \mathrm{p}=0.013]$; Figure 1). The treatment effect on body weight was similar in the mITT population: $-3.2 \mathrm{~kg}(-5.1,-1.2$; $\mathrm{p}=0.005) ;-2.7 \%(-4.3,-1.0 ; \mathrm{p}=0.006)$. In the beloranib group, significant weight loss was observed at Week 4 and Week 8, while the placebo group exhibited weight loss only during the 4-week OLE period when they received beloranib

167 (Figure 1). At Week 8, patients randomized to beloranib (PP population) exhibited LS mean (95\% CI)

168 weight loss of $-6.2 \mathrm{~kg}[-8.2,-4.1](-5.0 \%[-6.6,-3.3])$. Patients originally randomized to placebo 169 (placebo/beloranib) had weight loss of $-3.0 \mathrm{~kg}[-5.9,-0.1](-2.5 \%[-4.9,-0.1])$ from the start of beloranib 
treatment in the OLE, which was similar in magnitude to that observed during the first 4 weeks of

171 treatment in patients randomized to beloranib.

The single subject with a disease history of pituitary macroadenoma was randomized to placebo,

173 but exhibited a $1.8 \mathrm{~kg}(1.5 \%)$ mean weight loss during the OLE after starting beloranib treatment. The 1

174 placebo subject who completed the trial, but was not included in the PP analysis (dosing error) exhibited

$1750.0 \mathrm{~kg}$ difference from baseline at Week 4 , and $-3.3 \mathrm{~kg}$ weight change after 4 weeks of treatment with

176 beloranib, which was similar to that observed in placebo patients included in the PP analysis.

177 Patients with diabetes

178 Two patients with T2D were randomized to beloranib and exhibited weight loss consistent with the

179 overall cohort (-3.2 $\mathrm{kg}[-2.4 \%]$ and $-3.9 \mathrm{~kg}[-2.8 \%]$ at the end of the randomized period), whereas the

180 single placebo patient with T2D exhibited no weight change during the randomized period. One patient

181 with T2D did not complete the baseline HbA1c assessment. The remaining patient with T2D in the

182 beloranib group had an improvement in $\mathrm{HbAlc}$ from $8.5 \%$ (baseline) to $7.3 \%$ at 4 weeks and $6.6 \%$ at 8

183 weeks. The patient with T2D in the placebo group had a baseline HbA1c of 7\% was $6.7 \%$ at 4 weeks

184 (placebo) and 6.1\% at 8 weeks (beloranib).

Markers of Cardiometabolic Status

There was no change from baseline in total cholesterol, low-density lipoprotein, high-density

187 lipoprotein, and triglycerides at the end of this 4-week treatment period (Supplemental Table 2). Highsensitivity CRP (hsCRP) was highly elevated at baseline in these patients (mean [standard error]: 19.9

$189[3.9] \mathrm{mg} / \mathrm{L}$ for beloranib-randomized patients, 14.4 [6.1] for placebo-randomized patients; normal range 190 0-3 mg/L). hsCRP improved rapidly at the onset of beloranib treatment (Figure 2), and placebo patients 191 experienced a similar magnitude of reduction following initiation of beloranib in the OLE. At the follow192 up 7 days after cessation of study drug treatment, hsCRP concentrations returned to near baseline levels. 193 An exploratory analysis of additional biomarkers related to cardiometabolic health was also conducted 194 (Supplemental Table 2). 
Adiponectin and leptin, hormones related to metabolic dysregulation with obesity, were

197 significantly improved compared to placebo after 4 weeks of treatment. At the end of randomized

198 treatment, patients treated with beloranib exhibited significantly greater change from baseline in both

199 adiponectin (LS mean difference, $+1.2 \mathrm{mg} / \mathrm{L} ; \mathrm{p}<0.05)$ and leptin $(-19.8 \mu \mathrm{g} / \mathrm{L} ; \mathrm{p}<0.01$; Supplemental Table

200 2) compared with placebo. Beloranib-randomized patients appeared to maintain improvements through

201 the end of the extension period; placebo-randomized patients exhibited improvements in mean

202 adiponectin and leptin during the OLE that were similar in magnitude to those observed by beloranib-

203 treated patients during the randomized period (data not shown).

204 Reported Eating Behavior

205 There were no significant differences in change from baseline to the end of the randomized

206 treatment period between beloranib-treated vs. placebo-treated patients for any of the 8 items assessed

207 using the VAS questionnaire in this small subject population over 4 weeks. In the OLE period,

208 prospective food intake indicated favorable mean changes in both treatment arms: $-37 \%$ (95\%

$209 \mathrm{CI}:-53 \%,-21 \%)$ for the beloranib group and $-54 \%(-79 \%,-29 \%)$ following beloranib treatment of the

210 placebo-randomized patients.

211 Safety

212 During the randomized treatment period, the most frequent AEs were dizziness, headache, and

213 nasopharyngitis, Of these, dizziness was the only event reported with beloranib alone (Supplemental

214 Table 3). One patient in the placebo group withdrew from the study due to a treatment-emergent AE of

215 urticaria at the injection site that occurred following administration of study drug on study day 7; no

216 patients randomized to beloranib discontinued treatment due to an $\mathrm{AE}$ in the randomized treatment

217 period. The majority of events were mild in intensity ( $85 \%$ of all AEs) and transient, and there were no

218 severe events in the randomized period. 
220 period, AEs were primarily mild in intensity. One severe AE of back pain occurred during the OLE in a

221 patient randomized to beloranib; the Investigator did not attribute this to the study drug. During the OLE,

222 there were no AEs leading to discontinuation of study drug.

There were no serious AEs in the study, and there were no clinically meaningful or unexplained

224 changes in vital signs, electrocardiogram measurements, or clinical chemistry measurements. One

225 beloranib-treated subject experienced alanine aminotransferase (ALT) e 3 times the upper limit of normal

226 (ULN) and aspartate aminotransferase (AST) e 5 times the ULN, concurrent with the AEs of viral

227 gastroenteritis and fever. This patient was diagnosed with mild diarrhea/viral gastroenteritis that was

228 subsequently treated with anti-motility medications. ALT and AST levels returned to normal and the

229 patient continued in the study without any subsequent liver function test abnormalities. Two patients

230 randomized to beloranib experienced reduced neutrophil counts during the randomized period; both

231 events resolved during the OLE. One placebo-randomized patient experienced decreased neutrophil count

232 on the last day of beloranib treatment in the OLE, which was mild in intensity and resolved at follow-up.

233 None of these patients reported any AEs consistent with immunosuppression. Assessments of

234 coagulation (activated partial thromboplastin time, prothrombin time, International Normalized Ratio)

235 revealed no treatment differences, and there were no events related to venous thrombotic events.

237 Quality Questionnaire (median increase: +5.0 minutes) with beloranib at Week 2 that subsequently

238 returned to baseline at the next visit approximately 2 weeks later. There was a similar trend for patients

239 previously randomized to placebo (median increase: +5.0 minutes) in the second week of the OLE that

240 was maintained through the end of the OLE. 
In this randomized, double-blind, placebo-controlled Phase 2a study of beloranib in patients with

244 HIAO, beloranib produced statistically and clinically significant weight loss comparable to that observed

245 with beloranib in prior trials in neuroanatomically intact obese patients [9]. Beloranib was associated with

246 greater than $6 \mathrm{~kg}$ weight loss from baseline after to 8 weeks in patients with HIAO. This was observed

247 without diet and exercise counseling and without indication of a plateau in weight loss through 8 weeks.

248 HIAO is one of the most recalcitrant forms of obesity, so it is notable that the weight loss observed with

249 beloranib treatment in HIAO patients was comparable to that observed with beloranib in patients with

250 exogenous obesity [9]. This suggests that beloranib has potent effects on body weight that occur via

251 mechanisms that operate in the setting of compromised hypothalamic regulation of energy balance. The

252 patients in this study had long-standing HIAO with average onset of disease occurring a decade prior to

253 enrollment; typically a group considered refractory to treatment [16].

254 Mean weight change in HIAO after bariatric surgery peaks at $\sim 20 \%$ in the first year after Roux-

255 en-Y gastric bypass (RYGB) or sleeve gastrectomy [6], significantly less than the 35-40\% weight loss

256 typical for RYGB in patients with exogenous obesity [17]. Some effects of bariatric surgery are likely

257 mediated through the hypothalamus, perhaps accounting for the decreased efficacy in HIAO. The similar

258 weight loss observed in HIAO and exogenous obesity with beloranib treatment, albeit after 8 weeks,

259 suggests that a primary mechanism of weight loss associated with beloranib is independent of a fully

260 functioning hypothalamus. Additional studies in HIAO, including mechanistic studies and studies of

261 longer duration are required to fully characterize the effect of MetAP2 inhibition in HIAO.

Rapid improvement was observed in other biomarkers of inflammation, fatty liver disease,

263 insulin resistance, and other indicators of cardiovascular risk (Supplemental Table 2). In particular,

264 hsCRP was extremely elevated at baseline in patients with HIAO (mean $19.9 \mathrm{mg} / \mathrm{L}$ ), $>3$ times higher than

265 that observed in patients with other chronic diseases such as exogenous obesity, T2D, and polycystic

266 ovary syndrome [18-23]. Elevated hsCRP is associated with development of cardiovascular disease and 
267 diabetes [24, 25]. hsCRP levels fell rapidly at the onset of beloranib treatment (Figure 2) followed by a 268 rapid rise after cessation of treatment, suggesting that beloranib may exert salutary effects on hsCRP that 269 are independent of weight loss.

The mechanisms for the weight-independent effects of MetAP2 inhibition are under investigation.

271 One hypothesized mechanism for reduced hsCRP with MetAP2 inhibition is via suppression of

272 extracellular signal regulated kinase (ERK) hyperphosphorylation [26]. Putative ERK-mediated effects of

273 MetAP2 inhibition that influence inflammation, lipid biosynthesis, and insulin action include suppression

274 of sterol regulatory element binding protein (SREBP) activity [11, 12] and activation of retinoic acid-

275 related orphan receptor $\pm(\mathrm{ROR} \pm)[27]$.

276 The weight reduction observed with beloranib in patients with exogenous obesity may be

277 predominantly a result of reduced fat biosynthesis and increased fat oxidation and lipolysis, resulting in

278 reduced adipocyte mass as well as a reduction in measures of hunger and prospective food intake [9-12].

279 Although the short duration of this study makes it difficult to elucidate the mechanisms responsible for

280 weight loss in HIAO, the trend toward a reduction in prospective food intake observed in this study, in the

281 context of the underlying structural abnormalities of the hypothalamus in these patients, suggest

282 extrahypothalamic actions of appetite regulation mediated by beloranib treatment.

In patients with exogenous obesity treated with beloranib, the most commonly reported AEs were

284 insomnia and sleep disorders, followed by gastrointestinal disorders [9]. In patients with HIAO, beloranib

285 was not associated with a sustained increased incidence of insomnia and sleep disorders or

286 gastrointestinal AEs. A possible explanation for the apparent difference in AE pattern is that the medial

287 hypothalamus mediates the effects of beloranib on insomnia, sleep disorders, and gastrointestinal AEs.

288 Thus, patients with HIAO may not experience these AEs to the same degree, if at all. Furthermore,

289 beloranib may have simply attenuated the increased somnolence frequently observed in patients with

290 HIAO [28]. After conclusion of this study, 2 ongoing studies (1 in patients with Prader-Willi syndrome

291 and another in patients with exogenous obesity and T2D) were concluded early as a result of a complete 
292 clinical hold due to an imbalance in venous thromboembolic events, including 2 patient deaths due to

293 pulmonary embolism, in the beloranib development program. No venous thromboembolic events were

294 reported in the current trial; investigation is underway to elucidate a mechanism for the observed

295 imbalance in venous thromboembolic events.

This small proof of concept study was designed to provide initial data on the safety and efficacy

297 of beloranib in patients with HIAO. A limitation is our inability to precisely define the extent of

298 hypothalamic damage as patients were up to 25 years post-diagnosis and uniform MRI imaging was not

299 available. However, weight loss was generally consistent, implying that the location of hypothalamic

300 injury did not dramatically affect the response to treatment with a MetAP2 inhibitor. Improvements in

301 lipids occurred with beloranib treatment in other clinical studies of beloranib in patients with obesity [9,

302 13]. In this study, the small number of subjects and short duration of treatment likely contributed to the

303 lack of a statistically significant change in lipids. Additional studies of longer duration and larger sample

304 size are needed to obtain a better understanding of the effects of beloranib treatment.

305 This is the first study to demonstrate rapid and significant weight loss with beloranib, a MetAP2

306 inhibitor, in patients with HIAO. These results support a novel extra-hypothalamic mode of action of

307 beloranib to mediate improvements in weight and markers of adipocyte function and cardiovascular risk.

308 Given the impact on the severe obesity observed in patients with HIAO, MetAP2 inhibitors represent a

309 potential novel non-surgical treatment that warrants further investigation for not only HIAO, but as a

310 potential therapy for other causes of obesity without adequate efficacious therapies. Development of

311 second generation MetAP2 inhibitors is underway.

312

313 ACKNOWLEDGEMENTS

314 We thank Sonja K Billes, $\mathrm{PhD}$, and Brandon Walsh, $\mathrm{PhD}$, for medical writing assistance.

315 This work was funded by Zafgen, Inc. 


\section{AUTHOR CONTRIBUTIONS}

318 JP, MJA, TM and AHS participated as trial Investigators, discussion and interpretation of results, and 319 reviewed/edited manuscript. JM participated in conduct of the study, design and conduct of the statistical 320 analyses, discussion and interpretation of results, and reviewed/edited manuscript. DK participated in

321 design of study, conduct of study, discussion and interpretation of results, and reviewed/edited

322 manuscript. All authors approved the final manuscript.

323

324 FinANCIAL Disclosure

325 This study was funded by Zafgen, Inc.

326

327 CONFLICT OF INTEREST STATEMENT

328 JP, MJA and TM have nothing to declare. AHS is a consultant for Zafgen, Inc. and has received an 329 Investigator initiated grant from AstraZeneca. JM and DK are employed by Zafgen, Inc. 
332 [1] Muller HL, Emser A, Faldum A, et al. Longitudinal study on growth and body mass index before 333 and after diagnosis of childhood craniopharyngioma. J Clin Endocrinol Metab. 2004; 89: 3298-3305

334 [2] Muller HL. Craniopharyngioma. Endocr Rev. 2014; 35: 513-543

335 [3] Pereira AM, Schmid EM, Schutte PJ, et al. High prevalence of long-term cardiovascular, neurological and psychosocial morbidity after treatment for craniopharyngioma. Clin Endocrinol (Oxf). 2005; 62: 197-204

[4] Lustig RH. Hypothalamic obesity: causes, consequences, treatment. Pediatric endocrinology reviews : PER. 2008; 6: 220-227

[5] Lee M, Korner J. Review of physiology, clinical manifestations, and management of hypothalamic obesity in humans. Pituitary. 2009; 12: 87-95

[6] Bretault M, Boillot A, Muzard L, et al. Clinical review: Bariatric surgery following treatment for craniopharyngioma: a systematic review and individual-level data meta-analysis. J Clin Endocrinol Metab. 2013; 98: 2239-2246

[7] Gatta B, Nunes ML, Bailacq-Auder C, Etchechoury L, Collet D, Tabarin A. Is bariatric surgery really inefficient in hypothalamic obesity? Clin Endocrinol (Oxf). 2013; 78: 636-638

[8] Kim YM, An JJ, Jin YJ, et al. Assessment of the anti-obesity effects of the TNP-470 analog, CKD732. J Mol Endocrinol. 2007; 38: 455-465

[9] Kim DD, Krishnarajah J, Lillioja S, et al. Efficacy and safety of beloranib for weight loss in obese adults: a randomized controlled trial. Diabetes Obes Metab. 2015; 17: 566-572

[10] Rupnick MA, Panigrahy D, Zhang CY, et al. Adipose tissue mass can be regulated through the vasculature. Proc Natl Acad Sci U S A. 2002; 99: 10730-10735

[11] Kotzka J, Knebel B, Avci H, et al. Phosphorylation of sterol regulatory element-binding protein (SREBP)-1a links growth hormone action to lipid metabolism in hepatocytes. Atherosclerosis. 2010; 213: 156-165

[12] Raghow R, Yellaturu C, Deng X, Park EA, Elam MB. SREBPs: the crossroads of physiological and pathological lipid homeostasis. Trends Endocrinol Metab. 2008; 19: 65-73

[13] Hughes TE, Kim DD, Marjason J, Proietto J, Whitehead JP, Vath JE. Ascending dose-controlled trial of beloranib, a novel obesity treatment for safety, tolerability, and weight loss in obese women. Obesity (Silver Spring). 2013; 21: 1782-1788

[14] Flint A, Raben A, Blundell JE, Astrup A. Reproducibility, power and validity of visual analogue scales in assessment of appetite sensations in single test meal studies. Int J Obes Relat Metab Disord. 2000; 24: 38-48

[15] Kolotkin RL, Crosby RD, Kosloski KD, Williams GR. Development of a brief measure to assess quality of life in obesity. Obes Res. 2001; 9: 102-111

[16] Fothergill E, Guo J, Howard L, et al. Persistent metabolic adaptation 6 years after "The Biggest Loser" competition. Obesity (Silver Spring). 2016; 24: 1612-1619

[17] Sjostrom L, Lindroos AK, Peltonen M, et al. Lifestyle, diabetes, and cardiovascular risk factors 10 years after bariatric surgery. N Engl J Med. 2004; 351: 2683-2693

[18] Tan BK, Chen J, Hu J, et al. Metformin increases the novel adipokine cartonectin/CTRP3 in women with polycystic ovary syndrome. J Clin Endocrinol Metab. 2013; 98: E1891-1900

[19] Chan DC, Watts GF, Barrett PH, Beilin LJ, Mori TA. Effect of atorvastatin and fish oil on plasma high-sensitivity C-reactive protein concentrations in individuals with visceral obesity. Clin Chem. 2002; 48: 877-883

[20] Noureldein MH, Abd El-Razek RS, El-Hefnawy MH, El-Mesallamy HO. Fenofibrate reduces inflammation in obese patients with or without type 2 diabetes mellitus via sirtuin $1 /$ fetuin $A$ axis. Diabetes Res Clin Pract. 2015; 109: 513-520 
[21] Qian W, Zhu T, Tang B, et al. Decreased circulating levels of oxytocin in obesity and newly diagnosed type 2 diabetic patients. J Clin Endocrinol Metab. 2014; 99: 4683-4689

[22] Tan BK, Adya R, Farhatullah S, Chen J, Lehnert H, Randeva HS. Metformin treatment may increase omentin-1 levels in women with polycystic ovary syndrome. Diabetes. 2010; 59: 3023-3031 [23] Kip KE, Marroquin OC, Kelley DE, et al. Clinical importance of obesity versus the metabolic syndrome in cardiovascular risk in women: a report from the Women's Ischemia Syndrome Evaluation (WISE) study. Circulation. 2004; 109: 706-713

[24] Seven E, Husemoen LL, Sehested TS, et al. Adipocytokines, C-reactive protein, and cardiovascular disease: a population-based prospective study. PLoS One. 2015; 10: e0128987 [25] Pepys MB, Hirschfield GM. C-reactive protein: a critical update. J Clin Invest. 2003; 111: 18051812

[26] Datta B, Majumdar A, Datta R, Balusu R. Treatment of cells with the angiogenic inhibitor fumagillin results in increased stability of eukaryotic initiation factor 2-associated glycoprotein, p67, and reduced phosphorylation of extracellular signal-regulated kinases. Biochemistry. 2004; 43: 14821-14831 [27] Jetten AM, Kang HS, Takeda Y. Retinoic acid-related orphan receptors alpha and gamma: key regulators of lipid/glucose metabolism, inflammation, and insulin sensitivity. Front Endocrinol

(Lausanne). 2013; 4: 1

395 [28] Crowley RK, Woods C, Fleming M, et al. Somnolence in adult craniopharyngioma patients is a 
Table 1. Patient Demographics and Characteristics at Screening

\begin{tabular}{|c|c|c|}
\hline 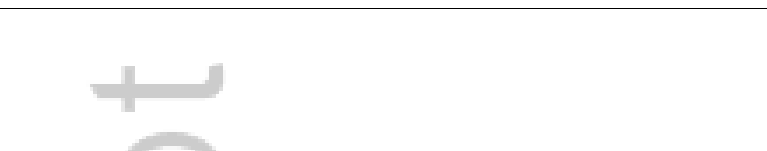 & $\begin{array}{l}\text { Placebo } \\
(\mathrm{N}=6)\end{array}$ & $\begin{array}{l}\text { Beloranib } \\
(\mathrm{N}=8)\end{array}$ \\
\hline Female, $\mathrm{n}(\%)$ & $4(66.7 \%)$ & $5(62.5 \%)$ \\
\hline Age, years & $34.3 \pm 8.8$ & $30.0 \pm 9.5$ \\
\hline Body weight, $\mathrm{kg}$ & $124.8 \pm 23.0$ & $127.6 \pm 22.8$ \\
\hline BMI, $\mathrm{kg} / \mathrm{m}^{2}$ & $42.1 \pm 6.0$ & $43.4 \pm 8.0$ \\
\hline White race, $\mathrm{n}(\%)$ & $4(66.7 \%)$ & $7(87.5 \%)$ \\
\hline Non-Hispanic/non-Latino, n (\%) & $5(83.3 \%)$ & $7(87.5 \%)$ \\
\hline \multicolumn{3}{|l|}{ Etiology of Hypothalamic Injury, n (\%) } \\
\hline Craniopharyngioma & $5(83.3 \%)$ & $8(100.0 \%)$ \\
\hline Pituitary macroadenoma & $1(16.7 \%)$ & 0 \\
\hline Time from confirmed hypothalamic injury, years & $11.4 \pm 9.4$ & $9.0 \pm 10.1$ \\
\hline \multicolumn{3}{|l|}{ Hormone replacement } \\
\hline Diabetes insipidus & $5(83.3 \%)$ & $8(100 \%)$ \\
\hline Central hypothyroidism & $6(100 \%)$ & $8(100 \%)$ \\
\hline Gonadotropin deficiency & $5(83.3 \%)$ & $6(75 \%)$ \\
\hline Adrenal insufficiency & $5(83.3 \%)$ & $7(87.5 \%)$ \\
\hline
\end{tabular}

400

401 Safety Population. Data are mean \pm SD except where indicated. 
403 FIGURE LEGENDS

404 Figure 1. Change in Body Weight

405 Data are least squares mean (SE) for the Per Protocol Population. The follow-up visit was 5-10 days after 406 the last dose of study drug. $\mathrm{BL}=$ baseline.

407 Figure 2. Change in hsCRP

408 Data are least squares mean (SE) for the Per Protocol Population. The follow-up visit was 5-10 days after 409 the last dose of study drug. $\mathrm{BL}=$ baseline.

This article is protected by copyright. All rights reserved. 


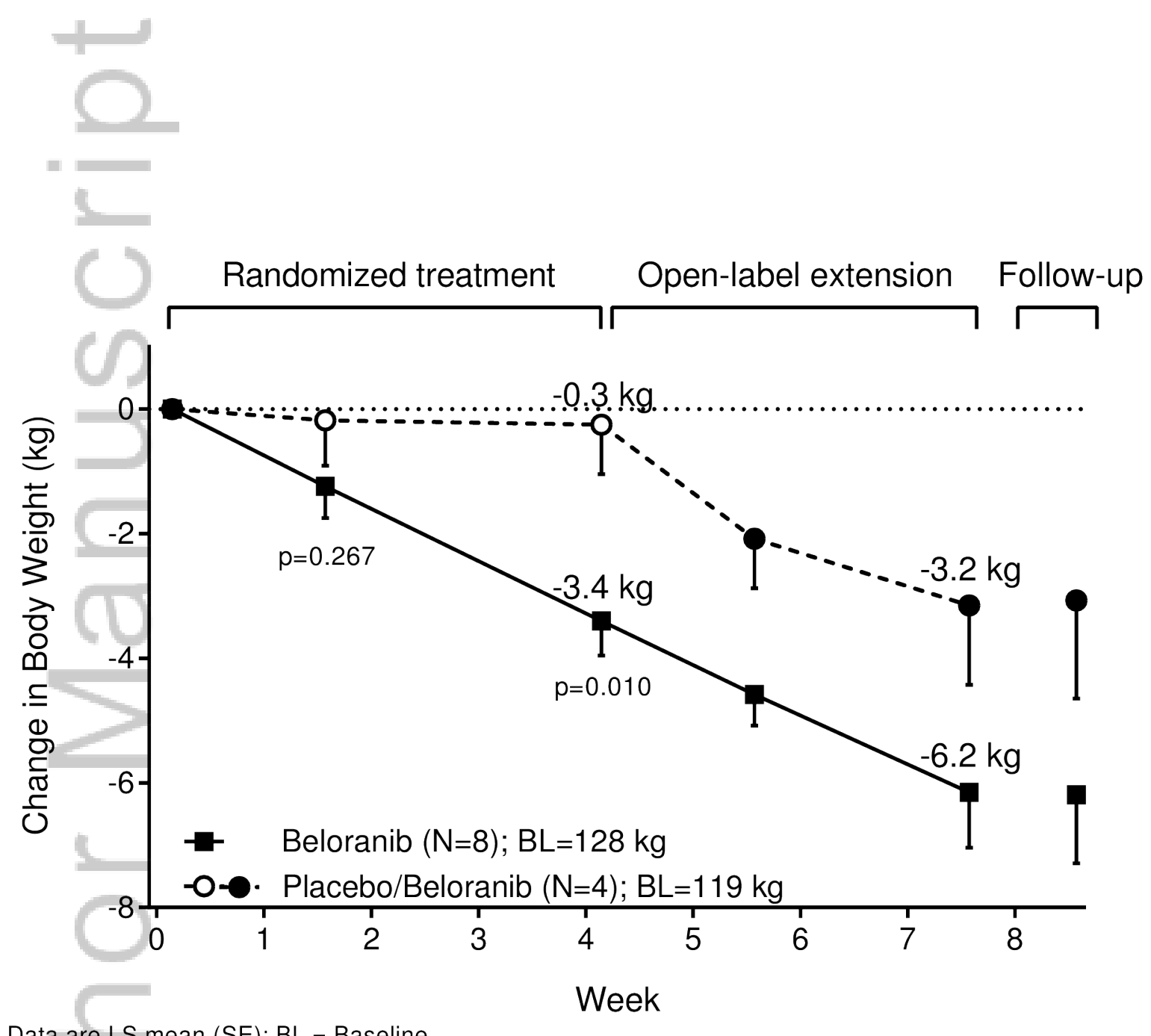

Data are LS mean (SE); BL = Baseline

dom-16-0848-op-file002.eps 


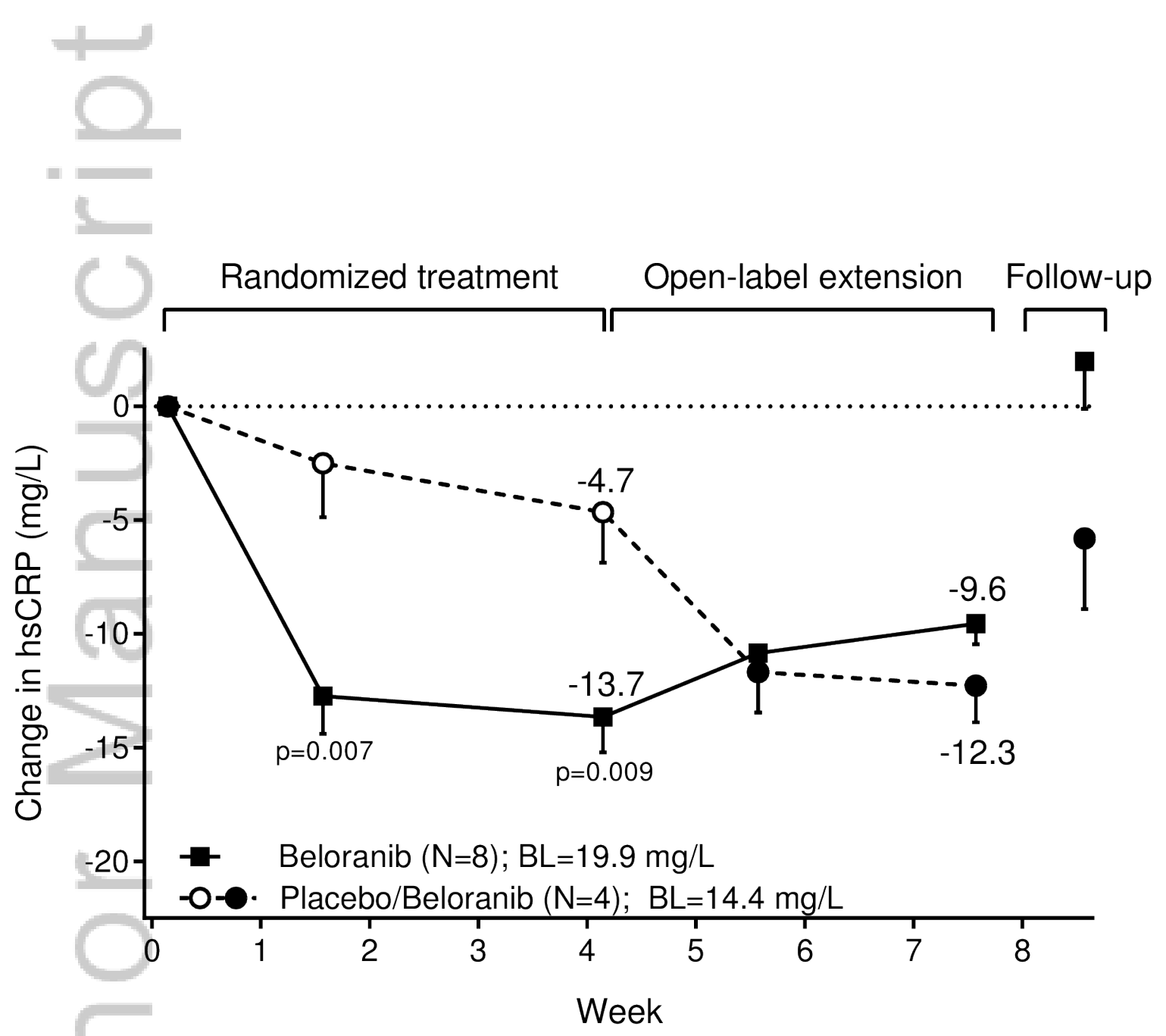

Data are LS mean (SE); BL = Baseline dom-16-0848-op-file003.eps 


\section{University Library}

\section{- M M N E R VA A gateway to Melbourne's research publications}

Minerva Access is the Institutional Repository of The University of Melbourne

Author/s:

Shoemaker, A;Proietto, J;Abuzzahab, MJ;Markovic, T;Malloy, J;Kim, DD

Title:

A randomized, placebo-controlled trial of beloranib for the treatment of hypothalamic injuryassociated obesity

Date:

2017-08-01

Citation:

Shoemaker, A., Proietto, J., Abuzzahab, M. J., Markovic, T., Malloy, J. \& Kim, D. D. (2017). A randomized, placebo-controlled trial of beloranib for the treatment of hypothalamic injuryassociated obesity. DIABETES OBESITY \& METABOLISM, 19 (8), pp.1165-1170. https:// doi.org/10.1111/dom.12928.

Persistent Link:

http://hdl.handle.net/11343/292775 